dimensional curvature ${ }^{7}$ and why curvaturetuned receptive fields apparently exist in the visual system ${ }^{8}$. In the absence of direct comparisons of their efficiency, however, it would be premature to conclude that the mechanisms of two-dimensional and threedimensional curvature discrimination are the same. Earlier suggestions that precise two-dimensional shape judgements evolved for the purposes of stereopsis do not withstand critical analysis".

A final thought on stereopsis is that it is likely to have several functions, including precise eye-hand coordination. The binocular reader who doubts the biological importance of stereoscopic information is invited to try collecting blackberries with one eye shut. The decreased performance level will be measured both by the decreased yield of fruit and the increased number of scratches. In normal reaching behaviour we have binocular vision not only of the object we are reaching for, but also of the hand. In these circumstances, it is not clear that the primary goal of stereopsis should be the extraction of absolute rather than relative distance information. Extraction of shape may be an additional bonus of stereopsis, rather than its primary function.

Michael J. Morgan is Professor of Psychology at University College London, Gower Street, London WC1E 6BT, UK.

\title{
Meteorites large and small
}

\section{D.E. Brownlee}

INTERPLANETARY dust particles and meteorites yield invaluable insights into the processes, materials and environments that existed during the earliest history of the Solar System. Conventional meteorites are wonderful samples but they provide an incomplete perspective of the comets and asteroids that have survived as relic planetary building materials. Dust grains, by contrast, provide a wider range of origins. On page 126 of this issue $^{1}$, Klöck et al. report analyses of unusual manganese-rich olivine and pyroxene grains that provide an interesting means of exploring possible links between materials in conventional meteorites and interplanetary dust particles that may be of cometary origin.

As recently discussed by R.T. Dodd in News and Views ${ }^{2}$, the thousands of conventional meteorites are probably fragments of only a small number of asteroids with no cometary representatives. Spectral-reflectance studies indicate that meteorites are not representative of typical asteroids nor do they reflect the range of materials seen in the asteroid belt. For example, the primitive 'D-class' objects that are abundant among the outer asteroids are composed of dark anhydrous materials $^{3}$ that have no meteoritic analogues. Selection effects associated with gravitational perturbation to Earthcrossing orbits and the strength required to survive atmospheric entry have produced a strong bias that has apparently prevented all comets and most asteroids from producing conventional meteorites.

In contrast, interplanetary dust particles (IDPs) potentially are derived from a broad range of both asteroids and shortperiod comets. Typical IDPs collected from the stratosphere are $5-50 \mu \mathrm{m}$ in size and such small particles are not significantly affected by the orbital and strength constraints imposed on conventional meteorites. Dust particles do not have to be strong to survive aerodynamic deceleration and, in space, the drag component of light pressure causes the orbits of all asteroid and cometary dust to spiral towards Earth-crossing orbits.

Using a transmission electron microscope, Klöck et al. ${ }^{1}$ have performed minorelement analyses on small olivine and pyroxene crystals contained in $0.1 \mu \mathrm{m}$ thick microtome sections cut from individual chondritic-composition IDPs only $10 \mu \mathrm{m}$ in size. Over half of the particles studied contained forsterite and enstatite grains with unusually high $\mathrm{MnO}$ contents above 0.5 per cent. The highest $\mathrm{MnO}$ content was 5 per cent with a $\mathrm{Mn} / \mathrm{Fe}$ ratio enhanced above the solar ratio by a factor of over 200. The authors name these unusual grains LIME (low-iron, manganese-enriched) because they are distinctly more Mn-rich than their (subLIME) counterparts commonly found in chondrites $^{4}$. In the meteorites examined, abundant LIME grains were found only in the fine-grained matrix of the Semarkona unequilibrated ordinary chondrite.

Similarities between this chondrite and a class of IDPs are of particular interest because Semarkona is one of the least metamorphosed ordinary chondrites and it contains smectites, carbides ${ }^{5}$ and enhanced deuterium in abundance as do some classes of interplanetary dust. The LIME grains as well as other minor- and trace-element anomalies in meteorite and IDP forsterite grains ${ }^{4}$ provide interesting ways of comparing primitive Solar System materials, but it is not currently possible uniquely to interpret the meaning of similarities. If components such as the LIME grains are produced only in a specific region of the solar nebula then their presence in both cometary and asteroidal materials would be evidence for mixing over a large radial distance scale. If radial mixing is not important and the LIME grains are specific to a process, such as condensation, then the process apparently occured over a wide radial range. Klöck et al. ${ }^{1}$ suggest that condensation is a probable origin for the LIME grains because it provides a means of decoupling the incorporation of $\mathrm{Fe}$ and Mn into the crystals and enhancing the $\mathrm{Mn} / \mathrm{Fe}$ ratio. Manganese can be stably incorporated into forsterite at a temperature only a few hundred degrees below the forsterite condensation temperature. Iron, on the other hand, should condense as metal at these temperatures and be essentially excluded from silicates. The high $\mathrm{Mn}$ and variable $\mathrm{Mn} / \mathrm{Fe}$ ratios in LIME grains contrast the behaviour of $\mathrm{Fe}$ and $\mathrm{Mn}$ in igneous meteorites. In these meteorites, $\mathrm{Mn}$ and $\mathrm{Fe}$ are highly correlated because $\mathrm{Mn}$ freely substitutes for $\mathrm{Fe}$ owing to the similarity of the ionic radius of $\mathrm{Fe}^{2+}$ and $\mathrm{Mn}^{2+}$.

If some interplanetary dust particles could be positively identified as cometary then their properties, such as content of LIME grains, and the properties of asteroidal IDPs and conventional meteorites would provide constraints on regions of the solar nebula where asteroids and short-period comets formed. The asteroids formed in the transition zone between the terrestrial and giant planets; the short-period comets presumably formed an order-of-magnitude farther from the centre of the Solar System, either in the Uranus-Neptune region or in the 'Kuiper belt' just beyond the outer planets. The nature of nebular solids could vary considerably over this range because of the pressure and temperature gradient in the solar nebula and also because inductive heating of small planetismals may have had a strong radial dependence ${ }^{6}$. Additional variations could be due to differences in the survival efficiency of presolar interstellar grains. Although comet samples must exist in present collections of interplanetary dust, it has not yet been possible to positively identify them. Flynn ${ }^{7}$ and Sandford and Bradley $^{8}$ now suggest that velocitydependent effects associated with atmospheric entry could be used as possible criteria to distinguish comet and asteriod samples collected in the stratosphere.

D.E. Brownlee is in the Department of Astronomy, University of Washington, Seattle, Washington 98195, USA.

\footnotetext{
1. Klöck, W., Thomas, K.L., McKay, D.S. \&. Palme, H. Nature 339, 126-128 (1989)

2. Dodd, R.T. Nature 338, 296-297 (1989).

3. Lebofsky, L.A. et al. Icarus (in the press).

4. Steele, I.M. Lunar and planet. Sci. XX, 1055-1056 (1989).

5. Hutcheon, R., Alexander, C.E.O. \& Barber, D.J. Geochim, cosmochim. Acta 51, 1875-1882 (1987).

6. Lebofsky, L.A., Jones, T.J. \& Herbert, F, in Origin and Evolution of Planetary and Satellite Atmospheres (eds Atreya, S.K., Pollack, J.B. \& Matthews, M.S.) (University of Arizona Press; in the press).

7. Flynn, G.J. Icarus 77, 287-310 (1989)

8. Sandford, S.A. \& Bradley, J.P. Icarus (in the press).
} 